\title{
TREES WITH UNIQUE MINIMUM GLOBAL OFFENSIVE ALLIANCE
}

\author{
Mohamed Bouzefrane, ${ }^{1, *}$, Isma Bouchemakh ${ }^{2}$, Mohamed Zamime $^{3}$ And \\ NOUREDDINE IKHLEF-ESCHOUF ${ }^{4}$
}

\begin{abstract}
Let $G=(V, E)$ be a simple graph. A non-empty set $D \subseteq V$ is called a global offensive alliance if $D$ is a dominating set and for every vertex $v$ in $V-D,\left|N_{G}[v] \cap D\right| \geq\left|N_{G}[v]-D\right|$. The global offensive alliance number is the minimum cardinality of a global offensive alliance in $G$. In this paper, we give a constructive characterization of trees having a unique minimum global offensive alliance.
\end{abstract}

Mathematics Subject Classification. 05C69, $05 \mathrm{C} 70$.

Received July 18, 2018. Accepted February 19, 2020.

\section{INTRODUCTION}

In this paper, $G=(V, E)$ is a simple graph with vertex set $V=V(G)$ and edge set $E=E(G)$. The open neighborhood of a vertex $v \in V$ is $N_{G}(v)=\{u \in V \mid u v \in E\}$ and the closed neighborhood of $v$ is $N_{G}[v]=$ $N_{G}(v) \cup\{v\}$. The degree of a vertex $v \in V$, denoted by $d_{G}(v)$, is the cardinality of its open neighborhood. A vertex of degree one is called a leaf and its neighbor is called a support vertex. If $v$ is a support vertex of a tree $T$, then $L_{T}(v)$ will denote the set of the leaves adjacent to $v$, and let $l_{T}(v)=\left|L_{T}(v)\right|$. The set of leaves and support vertices of $T$ is denoted by $L(T)$ and $S(T)$, respectively. Further, let $l(T)=|L(T)|$. As usual, the path of order $n$ is denoted by $P_{n}$, and the star of order $n$ by $K_{1, n-1}$. A double star, denoted by $S_{p, q}$, is a tree obtained from $P_{2}$ by joining $p$ pendent edges to one end and $q$ pendent edges to other end of $P_{2}$. A subdivision of an edge $u v$ is obtained by introducing a new vertex $w$ and replacing the edge $u v$ by the edges $u w$ and $w v$. A subdivided star, denoted by $S S_{k}$, is a star $K_{1, k}$ such that each edge is subdivided exactly once. A wounded spider is the tree formed by subdividing $r$ edges of $K_{1, k}$, where $1 \leq r \leq k-1$. For a vertex $v$, in a rooted tree $T$, let $C(v)$ and $D(v)$ denote the set of children and descendants of $v$, respectively. Let $D[v]=D(v) \cup\{v\}$. The maximal subtree at $v$, denoted by $T_{v}$, is the subtree of $T$ induced by $D[v]$.

Keywords. Tree, domination, global offensive alliance, characterization.

1 Department of Mathematics, B.P. 270, Lamda-RO Laboratory, University of Blida, Blida, Algeria.

2 Faculty of Mathematics, Laboratory L'IFORCE, University of Sciences and Technology Houari Boumediene (USTHB), B.P. 32 El-Alia, Bab-Ezzouar 16111, Algiers, Algeria.

3 Faculty of Technology, University of Médéa, Médéa, Algeria.

4 Faculty of Sciences, Department of Mathematics and Computer Science, University of Médéa, Médéa, Algeria.

* Corresponding author: mohamedbouzefrane@gmail.com 
If $G$ and $H$ are two vertex-disjoint graphs, the union of $G$ and $H$ is the graph $G \cup H$ whose vertex-set is $V(G) \cup V(H)$ and edge-set is $E(G) \cup E(H)$. For an integer $k \geq 2$, the union of $k$ copies of graph $G$ is denoted $k G$ and the union of graphs $G_{1}, G_{2}, \ldots, G_{k}$ is denoted $\cup_{i=1}^{k} G_{i}$.

Let $D \subseteq V$ be a non-empty set of vertices of graph $G$. D is a dominating set of $G$ if every vertex in $V-D$ has at least one neighbor in $D$. The domination number of $G$, denoted by $\gamma(G)$, is the minimum cardinality of a dominating set of $G$. $D$ is an offensive alliance of $G$ if for every $v \in V-D$, we have

$$
\left|N_{G}[v] \cap D\right| \geq\left|N_{G}[v]-D\right| .
$$

$D$ is a global offensive alliance, or briefly a goa, of $G$ if $D$ is both an offensive alliance and a dominating set of $G$. The global offensive alliance number, denoted by $\gamma_{o}(G)$, is the minimum cardinality of a goa of $G$. Note that $\gamma_{o}$ is defined for every graph. For a graph $G$, the vertex set $V$ of $G$ is a goa, as $V-V=\emptyset$, and the condition for $V$ to be a goa is (vacuously) true. A goa of $G$ of cardinality $\gamma_{o}(G)$ is called a $\gamma_{o}(G)$-set. $G$ is a unique global offensive alliance graph, or briefly an ugoa-graph, if $G$ has a unique $\gamma_{o}(G)$-set. A unique global offensive alliance on a graph $G$ allows vertices to have a unique assignment. This can be used for an extension of the graph while keeping the same layout which existed.

The literature on the concept of domination and its variants has been surveyed and detailed in the books of Haynes et al. $[25,26]$. Among these variants, there are the alliances in graphs. The study of alliances in graphs is first investigated by Kristiansen et al. [31]. Favaron et al. [11] initiated and made a thorough study of offensive alliances, they derived several bounds on the offensive alliance number. Several other bounds on (global) offensive alliance number are obtained by Rodríguez-Velázquez and Sigarreta [34,35], Rad [33], Harutyunyan [22,23] and, Yero and Rodríguez-Velá zquez [38]. Concerning the computational complexity, the NP-completeness is proved by Balakrishnan et al. [1] for the problem of global offensive alliance and by Fernau et al. [12] for both problems (offensive and global offensive alliances) in general graphs. More results can be found in [3-6,10,32].

Graphs with a unique minimum $\mu$-set, where $\mu$ is some domination parameter, has attracted the interest of several researchers over the last decades. In particular, graphs with a unique minimum dominating sets have been studied, by Gunther et al. [21]. These authors initiated this related concept to uniqueness in the domination theory and investigated some of the structural properties of graphs having this property. Some works have been established on different classes of graphs, for example, by Fischermann and Volkmann [15] for trees and in [16] for cactus graphs, by Fischermann [13] for block graphs, by Hedetniemi [27] for some cartesian product graphs and in [28] for some repeated cartesian products graphs. Regarding the uniqueness related to other invariants of domination, studies have been undertaken by Blidia et al. [2] for the locating-domination number, by Chellali and Haynes for paired-domination number in [7] and for double domination number in [8], Chellali and Rad [9] for roman domination number, and by Haynes and Henning [24] for total domination number. Further works on this topic can be found in [14,17-20,29,30,36,37].

In this paper, we are interested in determining the structure of trees that are ugoa-trees. The paper is organized as follows. Section 2 deals with the preservation of the ugoa-tree property after having performed some operation on it. In Section 3, we provide a constructive characterization of ugoa-tree. Finally, we conclude by setting some problems that we seem interesting.

\section{Preliminary Results}

We first present some elementary facts concerning the ugoa-trees. Some results are straightforward and so their proofs are omitted.

Observation 2.1. Let $T$ be a tree of order at least three and let $u \in S(T)$. Then,

(i) there is a $\gamma_{o}(T)$-set that contains all support vertices of $T$,

(ii) if $D$ is a unique $\gamma_{o}(T)$-set, then $D$ contains all support vertices of $T$ but no leaf,

(iii) if $l_{T}(u) \geq 2$, then $u$ belongs to any $\gamma_{o}(T)$-set. 
Proof. (i) and (ii) are obvious. Let $S$ be any $\gamma_{o}(T)$-set and assume that item (iii) is not satisfied. In this case, all leaves adjacent to $u$ would be contained in $S$ and the set $\left(S-L_{T}(u)\right) \cup\{u\}$ would be a goa of $T$ of cardinality less than $\gamma_{o}(T)$, a contradiction.

Observation 2.2. Let $T$ be a tree obtained from a nontrivial tree $T^{\prime}$ by joining a new vertex $v$ at a support vertex $u$ of $T^{\prime}$. Let $D$ and $D^{\prime}$ be $\gamma_{o}(T)$-sets of $T$ and $T^{\prime}$, respectively. Then,

(i) $\left|D^{\prime}\right|=|D|$,

(ii) $D \cap V\left(T^{\prime}\right)$ is a $\gamma_{o}\left(T^{\prime}\right)$-set,

(iii) if $T$ is an ugoa-tree such that $u$ is in any $\gamma_{o}\left(T^{\prime}\right)$-set, then $T^{\prime}$ is an ugoa-tree.

Proof. According to Observation 2.1(iii), and since $l_{T}(u) \geq 2, u$ must be in $D$.

(i) $D$ is clearly a goa of $T^{\prime}$, and then $\left|D^{\prime}\right| \leq|D|$. By Observation 2.1(i) we can assume that $u \in D^{\prime}$. Hence, $D^{\prime}$ can be extended to a goa of $T$, which leads to $|D| \leq\left|D^{\prime}\right|$. Thus, equality holds.

(ii) Since $D \cap V\left(T^{\prime}\right)=D$ is a goa of $T^{\prime}$ of cardinality $|D|=\left|D^{\prime}\right|$, we deduce that $D \cap V\left(T^{\prime}\right)$ is a $\gamma_{o}\left(T^{\prime}\right)$-set.

(iii) Item (i) together with the fact that $u$ belongs to any $\gamma_{o}\left(T^{\prime}\right)$ imply that $D^{\prime}$ can be extended to a $\gamma_{o}(T)$-set. Therefore, the uniqueness of $D$ as a $\gamma_{o}(T)$-set leads to $D^{\prime}=D$. Hence, $D^{\prime}$ is the unique $\gamma_{o}\left(T^{\prime}\right)$.

Observation 2.3. Let $T$ be a tree obtained from a nontrivial tree $T^{\prime}$, different from $P_{2}$, by joining the center vertex $y$ of the path $P_{3}=x-y-z$ at a support vertex $v$ of $T^{\prime}$. Let $D$ and $D^{\prime}$ be $\gamma_{o}(T)$-sets of $T$ and $T^{\prime}$, respectively such that each of them contains all support vertices. Then,

(i) $\left|D^{\prime}\right|=|D|-1$,

(ii) $D \cap V\left(T^{\prime}\right)$ is a $\gamma_{o}\left(T^{\prime}\right)$-set,

(iii) if $T$ is an ugoa-tree, then $T^{\prime}$ is an ugoa-tree.

Proof. (i) Since $y \in D$ and $v \in D \cap D^{\prime}$, the set $D-\{y\}$ is a goa of $T^{\prime}$ and then $\left|D^{\prime}\right| \leq|D|-1$. Moreover, since $v \in D^{\prime}, D^{\prime}$ can be extended to a goa of $T$ by adding $y$. It follows, $|D| \leq\left|D^{\prime} \cup\{y\}\right|=\left|D^{\prime}\right|+1$ and equality holds.

(ii) Since $D \cap V\left(T^{\prime}\right)=D-\{y\}$ is a goa of $T^{\prime}$ of cardinality $|D|-1=\left|D^{\prime}\right|, D \cap V\left(T^{\prime}\right)$ is a $\gamma_{o}\left(T^{\prime}\right)$-set.

(iii) Let $B=\{y\}$. In view of item (i), $D^{\prime}$ can be extended to a $\gamma_{o}(T)$-set by adding the unique vertex of $B$. This and item (ii) together with the uniqueness of $D$ imply that $D^{\prime}=D \cap V\left(T^{\prime}\right)$ is the unique $\gamma_{o}\left(T^{\prime}\right)$-set.

Observation 2.4. Let $T$ be a tree obtained from a nontrivial tree $T^{\prime}$ by adding a subdivided star $S S_{k}$ with $k$ leaves, $k \geq 1$, and then identifying its center to some leaf $v$ of $T^{\prime}$. Let $w$ be the support vertex adjacent to $v$ in $T^{\prime}$, and let $D$ and $D^{\prime}$ be $\gamma_{o}(T)$-sets of $T$ and $T^{\prime}$, respectively. If $w \in D \cap D^{\prime}$, then the following three properties are satisfied.

(i) $\left|D^{\prime}\right|=|D|-k$,

(ii) $D \cap V\left(T^{\prime}\right)$ is a $\gamma_{o}\left(T^{\prime}\right)$-set,

(iii) if $T$ is an ugoa-tree, then $T^{\prime}$ is an ugoa-tree.

Proof. Let $B=\left\{y_{1}, y_{2}, \ldots, y_{k}\right\}$ be the set of support vertices of $S S_{k}$. Definition of $T$ implies that every vertex in $B$ is adjacent to $v$.

(i) Obviously, each vertex in $B$ remains a support vertices in $T$. Hence, in view of Observation 2.1(i), we can assume that $D$ contains all vertices of $B$. Therefore, since $w \in D$ and $v \notin D, D-B$ is a goa of $T^{\prime}$, and consequently, $\left|D^{\prime}\right| \leq|D-B|=|D|-k$. We now prove the opposite inequality. $w \in D^{\prime}$ implies that $D^{\prime}$ can be extended to a goa of $T$ by adding all vertices of $B$. Hence, $|D| \leq\left|D^{\prime} \cup B\right|=\left|D^{\prime}\right|+k$ and equality holds. 
(ii) The proof is similar to that of Observation 2.3(ii), by taking $D \cap V\left(T^{\prime}\right)=D-B$.

(iii) The proof is similar to that of Observation 2.3(iii).

Observation 2.5. Let $V\left(T^{\prime}\right)$ be the vertex set of a nontrivial tree $T^{\prime}$, and let $D^{\prime}$ be a $\gamma_{o}\left(T^{\prime}\right)$-set such that $V\left(T^{\prime}\right)-D^{\prime}$ has a vertex $w$ of degree $q \geq 2$ in $T^{\prime}$ and $\left|N_{T^{\prime}}(w) \cap\left(V\left(T^{\prime}\right)-D^{\prime}\right)\right| \leq 1$. Let $p$ be a positive integer such that

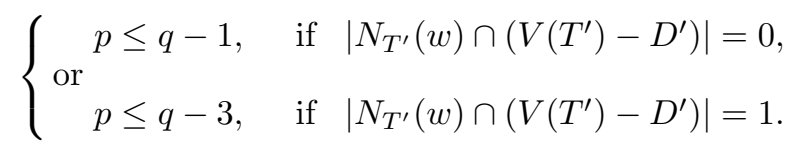

Let $T$ be a tree obtained from $T^{\prime}$ by adding $p$ subdivided stars $S S_{k_{1}}, \ldots, S S_{k_{p}}, k_{i} \geq 2$ for $1 \leq i \leq p$, and joining each center $x_{i}$ of $S S_{k_{i}}, 1 \leq i \leq p$, at $w$. Let $D$ be a $\gamma_{o}(T)$-set. If $w$ and $x_{1}, x_{2}, \ldots, x_{p}$ are not in $D$, then the following three properties are satisfied.

(i) $\left|D^{\prime}\right|=|D|-\sum_{i=1}^{p} k_{i}$,

(ii) $D \cap V\left(T^{\prime}\right)$ is a $\gamma_{o}\left(T^{\prime}\right)$-set,

(iii) if $T$ is an ugoa-tree, then $T^{\prime}$ is also an ugoa-tree.

Proof. For $i \in\{1, \ldots, p\}$, let $B_{i}$ the set of the support vertices of $S S_{k_{i}}$.

(i) Since the vertices $w, x_{1}, x_{2}, \ldots, x_{p}$ are not in $D$, all vertices of $\cup_{i=1}^{p} B_{i}$ must be in $D$. Therefore, $D-\cup_{i=1}^{p} B_{i}$ is a goa of $T^{\prime}$, giving that $\left|D^{\prime}\right| \leq|D|-\sum_{i=1}^{p} k_{i}$. For the opposite inequality, we first prove that the set $A=\cup_{i=1}^{p} B_{i} \cup D^{\prime}$ is a goa of $T$, that is, $\left|N_{T}[z] \cap A\right| \geq\left|N_{T}[z]-A\right|$ holds, for each $z \in\left\{w, x_{1}, x_{2}, \ldots, x_{p}\right\}$. For this, we consider two cases depending on the value of $z$.

Case 1. $z=x_{i}$, for some $i \in\{1, \ldots, p\}$.

We then have

$$
\left|N_{T}[z] \cap A\right|=\left|N_{T}[z] \cap\left(\cup_{i=1}^{p} B_{i}\right)\right|=k_{i} \geq 2,
$$

and

$$
\left|N_{T}[z]-A\right|=|\{z, w\}|=2 .
$$

Thus, $\left|N_{T}[z] \cap A\right| \geq\left|N_{T}[z]-A\right|$, for each $z \in\left\{x_{1}, x_{2}, \ldots, x_{p}\right\}$.

Case 2. $z=w$.

We have

$$
\left|N_{T}[z] \cap A\right|=\left\{\begin{array}{lll}
q, & \text { if } & \left|N_{T^{\prime}}(w) \cap\left(V\left(T^{\prime}\right)-D^{\prime}\right)\right|=0 \\
q-1, & \text { if } & \left|N_{T^{\prime}}(w) \cap\left(V\left(T^{\prime}\right)-D^{\prime}\right)\right|=1
\end{array}\right.
$$

and

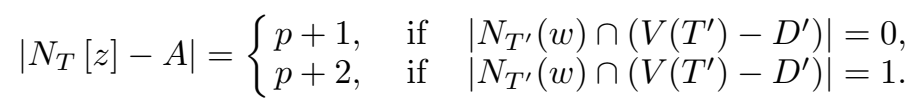

According to (2.1), we have in this case also, $\left|N_{T}[z] \cap A\right| \geq\left|N_{T}[z]-A\right|$, for $z=w$. Therefore, $A$ is a goa of $T$, and

$$
|D| \leq|A|=\left|D^{\prime}\right|+\sum_{i=1}^{p} k_{i} .
$$

Hence, the equality holds, as required.

(ii) Using the fact that $D \cap V\left(T^{\prime}\right)=D-\cup_{i=1}^{p} B_{i}$, the proof is similar to that of Observation 2.3(ii).

(iii) The proof is similar to that of Observation 2.3(iii), by taking $B=\cup_{i=1}^{p} B_{i}$. 


\section{The MAIn RESUlT}

In order to characterize the trees with a unique minimum global offensive alliance, we define a family $\mathcal{F}$ of all trees $T$ that can be obtained from a sequence $T_{1}, T_{2}, \ldots, T_{r}, r \geq 1$, of trees, where $T_{1}$ is the path $P_{3}$ centered at a vertex $y, T=T_{r}$, and, if $r \geq 2$, the tree $T_{i+1}$ is obtained from $T_{i}, 1 \leq i \leq r-1$, by one of the following operations listed below. Let $A\left(T_{1}\right)=\{y\}$.

- Operation $\mathcal{O}_{1}$ : Attach a vertex by joining it to any support vertex of $T_{i}$. Let $A\left(T_{i+1}\right)=A\left(T_{i}\right)$.

- Operation $\mathcal{O}_{2}$ : Attach a path $P_{3}=u$ - $v$-w by joining $v$ to any support vertex of $T_{i}$. Let $A\left(T_{i+1}\right)=A\left(T_{i}\right) \cup\{v\}$.

- Operation $\mathcal{O}_{3}$ : Adding a subdivided star $S S_{k}$ with $k$ leaves, $k \geq 1$, and then identifying the center of it to the leaf of $T_{i}$, where its support vertex $w$ in $T_{i}$ satisfies the following condition $C_{0}$.

$\left(C_{0}\right)$ : If $\left|N_{T_{i}}[w] \cap A\left(T_{i}\right)\right| \geq\left|N_{T_{i}}(w) \cap\left(V\left(T_{i}\right)-A\left(T_{i}\right)\right)\right|+1$, then

- either $l_{T_{i}}(w)=2$ and $N_{T_{i}}(w)-A\left(T_{i}\right)$ has a least one vertex $w_{t}$, such that $\left|N_{T_{i}}\left(w_{t}\right) \cap A\left(T_{i}\right)\right| \leq$ $\left|N_{T_{i}}\left[w_{t}\right] \cap\left(V\left(T_{i}\right)-A\left(T_{i}\right)\right)\right|+1$,

- or $l_{T_{i}}(w)=1$ and $N_{T_{i}}(w)-A\left(T_{i}\right)$ has two vertices $w_{p}$ and $w_{q}$, such that, $\left|N_{T_{i}}\left(w_{l}\right) \cap A\left(T_{i}\right)\right| \leq$ $\left|N_{T_{i}}\left[w_{l}\right] \cap\left(V\left(T_{i}\right)-A\left(T_{i}\right)\right)\right|+1$, for $l=p, q$.

Let $A\left(T_{i+1}\right)=A\left(T_{i}\right) \cup B$, where $B$ is the set of support vertices of $S S_{k}$.

- Operation $\mathcal{O}_{4}$ : Let $w \in V\left(T_{i}\right)-A\left(T_{i}\right)$ be a vertex of degree $q \geq 2$ in $T_{i}$, such that $\left|N_{T_{i}}(w) \cap\left(V\left(T_{i}\right)-A\left(T_{i}\right)\right)\right| \leq 1$. Attach $p \geq 1$ subdivided stars $S S_{k_{i}}, k_{i} \geq 2$ for $1 \leq i \leq p$, by joining each center $x_{i}$ of $S S_{k_{i}}$, at $w$, such that

$$
p \leq\left\{\begin{array}{l}
q-1 \text { if }\left|N_{T_{i}}(w) \cap\left(V\left(T_{i}\right)-A\left(T_{i}\right)\right)\right|=0, \\
q-3 \text { if }\left|N_{T_{i}}(w) \cap\left(V\left(T_{i}\right)-A\left(T_{i}\right)\right)\right|=1 .
\end{array}\right.
$$

Let $A\left(T_{i+1}\right)=A\left(T_{i}\right) \cup\left(\cup_{i=1}^{p} B_{i}\right.$, where $B_{i}$ is the set of support vertices of $S S_{k_{i}}$.

Before stating our main result, we need to prove an additional lemma.

Lemma 3.1. If $T \in \mathcal{F}$, then $A(T)$ is the unique $\gamma_{o}(T)$-set.

Proof. Let $T \in \mathcal{F}$. We proceed by induction on the number of operations, say $r$, required to construct $T$. The property is true if $r=1$, since $T=T_{1}$ is the path $P_{3}$ centered at $y$ and $A(T)=\{y\}$ is the unique $\gamma_{o}(T)$-set. This establishes the base case.

Assume that for any tree $T^{\prime} \in \mathcal{F}$ that can be constructed with $r-1$ operations from $T_{1}, A\left(T^{\prime}\right)$ is the unique $\gamma_{o}\left(T^{\prime}\right)$-set. Let $T=T_{r}$, with $r \geq 2$, and $T^{\prime}=T_{r-1}$. We consider four cases, depending on the used operation.

Case 1. $T$ is obtained from $T^{\prime}$ by using Operation $\mathcal{O}_{1}$.

Assume that $T$ is obtained from $T^{\prime}$ by attaching an extra vertex at a support vertex $u$ of $T^{\prime}$. In view of Observation 2.1(ii), $u \in A\left(T^{\prime}\right)$. Hence, $A\left(T^{\prime}\right)$ can be extended to a goa of $T$. By Observation 2.2(i), $\gamma_{o}(T)=$ $\gamma_{o}\left(T^{\prime}\right)$, and then $A\left(T^{\prime}\right)$ is a $\gamma_{o}(T)$-set. Since $A\left(T^{\prime}\right)$ is the unique $\gamma_{o}\left(T^{\prime}\right)$-set, we infer that $A(T)=A\left(T^{\prime}\right)$ is the unique $\gamma_{o}(T)$-set.

Case 2. $T$ is obtained from $T^{\prime}$ by using Operation $\mathcal{O}_{2}$.

$A\left(T^{\prime}\right) \cup\{v\}$ is a goa of $T$. By Observation 2.3(i), $\gamma_{o}(T)=\gamma_{o}\left(T^{\prime}\right)+1$, and then $A\left(T^{\prime}\right) \cup\{v\}$ is a $\gamma_{o}(T)$-set. Since $A\left(T^{\prime}\right)$ is the unique $\gamma_{o}\left(T^{\prime}\right)$-set, we infer that $A(T)=A\left(T^{\prime}\right) \cup\{v\}$ is the unique $\gamma_{o}(T)$-set.

Case 3. $T$ is obtained from $T^{\prime}$ by using Operation $\mathcal{O}_{3}$.

$A\left(T^{\prime}\right) \cup B$ is a goa of $T$. By Observation 2.4(i), $\gamma_{o}(T)=\gamma_{o}\left(T^{\prime}\right)+k$, and then $A\left(T^{\prime}\right) \cup B$ is a $\gamma_{o}(T)$-set. Since $A\left(T^{\prime}\right)$ is the unique $\gamma_{o}\left(T^{\prime}\right)$-set, we infer that, $A(T)=A\left(T^{\prime}\right) \cup B$ is the unique $\gamma_{o}(T)$-set.

Case 4. $T$ is obtained from $T^{\prime}$ by using Operation $\mathcal{O}_{4}$.

$A\left(T^{\prime}\right) \cup\left(\cup_{i=1}^{p} B_{i}\right)$ is a goa of $T$. By Observation 2.5(i), $\gamma_{o}(T)=\gamma_{o}\left(T^{\prime}\right)+\sum_{i=1}^{p} k_{i}$, and then $A\left(T^{\prime}\right) \cup\left(\cup_{i=1}^{p} B_{i}\right)$ is a $\gamma_{o}(T)$-set. Since $A\left(T^{\prime}\right)$ is the unique $\gamma_{o}\left(T^{\prime}\right)$-set, we infer that $A(T)=A\left(T^{\prime}\right) \cup\left(\cup_{i=1}^{p} B_{i}\right)$ is the unique $\gamma_{o}(T)$-set. 
Note that in each one of the previously four cases, $A\left(T_{i+1}\right)$ is obtained from $A\left(T_{i}\right)$ by adding all support vertices in $T_{i+1}-T_{i}$. Hence, the following corollary is immediate.

Corollary 3.2. Let $T \in \mathcal{F}$ and $S(T)$ be a set of support vertices in $T$. Then, $\gamma_{o}(T) \geqslant|S(T)|$.

We are now ready to prove our main result.

Theorem 3.3. A tree $T$ is an ugoa-tree if and only if $T=K_{1}$ or $T \in \mathcal{F}$.

Proof. It is obvious that $T=K_{1}$ is an ugoa-tree. Also, Lemma 3.1 states that any member of $\mathcal{F}$ is an ugoa-tree. We prove the converse by induction on the number $n$ of vertices of $T$. For $n=1$ or $n=3$, the result trivially holds, but not for $n=2$, since $P_{2}$ is not an ugoa-tree. For $n=4, T \in\left\{K_{1,3}, P_{4}\right\}$. Clearly $P_{4}$ is not an ugoa-tree, whilst $K_{1,3}$ is an ugoa-tree that can be obtained from a $P_{3}$ using Operation $\mathcal{O}_{1}$. Hence, $K_{1,3} \in \mathcal{F}$. For $n=5$, $T \in\left\{S_{1,2}, K_{1,4}, P_{5}\right\}$. The double star $S_{1,2}$ is not an ugoa-tree, while $K_{1,4}$ and $P_{5}$ are ugoa-trees, since $K_{1,4}$ can be obtained from $K_{1,3}$ by using Operation $\mathcal{O}_{1}$, and $P_{5}$ can be obtained from a $P_{3}$ by using Operation $\mathcal{O}_{3}$. Therefore, $K_{1,4}$ and $P_{5}$ are in $\mathcal{F}$. This establishes the base case.

Assume now $n \geq 6$, and any tree $T^{\prime}$ of order $n^{\prime}, 3 \leq n^{\prime}<n$, has a unique $\gamma_{o}\left(T^{\prime}\right)$-set in $\mathcal{F}$. Let $T$ be a tree of order $n$ with the unique $\gamma_{o}(T)$-set $D$ and let $s \in S(T)$. By Observation 2.1(ii), we have $s \in D$. If $l_{T}(s) \geq 3$, then let $T^{\prime}$ be the tree obtained from $T$ by removing a leaf adjacent to $s$ and let $D^{\prime}$ be a $\gamma_{o}\left(T^{\prime}\right)$-set. Then, clearly $n^{\prime}=\left|V\left(T^{\prime}\right)\right|=n-1 \geq 5, l_{T^{\prime}}(s) \geq 2$ and so, $s \in D^{\prime}$ by Observation 2.1(iii). According to Observation 2.2(iii), $T^{\prime}$ is an ugoa-tree. Applying the inductive hypothesis to $T^{\prime}$, we get $T^{\prime} \in \mathcal{F}$. Thus, $T$ is obtained from $T^{\prime}$ by Operation $\mathcal{O}_{1}$, implying that $T \in \mathcal{F}$. Assume now that

$$
\text { for each } x \in S(T), \quad l_{T}(x) \leq 2 .
$$

Root $T$ at a vertex $r$ of maximum eccentricity. Let $u$ be a support vertex of maximum distance from $r$ and let $u^{\prime}$ be a leaf adjacent to $u$. Let $v$ and $w$ be the parents of $u$ and $v$, respectively, in the rooted tree. We consider two cases.

Case 1. $v \in D$.

If $l_{T}(u)=1$, then $D \cup\left\{u^{\prime}\right\}-\{u\}$ is a $\gamma_{o}(T)$-set, contradicting the uniqueness of $D$ as a $\gamma_{o}(T)$-set. Hence, by $(3.1), l_{T}(u)=2$. We claim that $v \in S(T)$. Assume to the contrary that $v \notin S(T)$. Then, either $w \in D$ and so, $D-\{v\}$ is a goa of $T$ of cardinality less than $|D|$, contradicting the minimality of $D$, or $w \notin D$ and so, $D-\{v\} \cup\{w\}$ is a $\gamma_{o}(T)$-set, contradicting the uniqueness of $D$ as a $\gamma_{o}(T)$-set. This completes the proof of the claim. Let $T^{\prime}=T-T_{u}$ and $D^{\prime}$ be a $\gamma_{o}\left(T^{\prime}\right)$-set. By Observation 2.1(i), we can assume that $D^{\prime}$ contains all support vertices in $T^{\prime}$. Since $\left|V\left(T_{u}\right)\right|=3$, it follows that $n^{\prime}=\left|V\left(T^{\prime}\right)\right|=n-3 \geq 3$ and so, $T^{\prime} \neq P_{2}$. By Observation 2.3(iii), $T^{\prime}$ is an ugoa-tree. Applying our inductive hypothesis, we get $T^{\prime} \in \mathcal{F}$. Thus, $T$ can be obtained from $T^{\prime}$ by Operation $\mathcal{O}_{2}$ and so, $T \in \mathcal{F}$.

Case 2. $v \notin D$.

According to Observation 2.1(ii), $v \notin S(T)$ and then, $l_{T}(v)=0$. Let $k=\left|N_{T}(v)-\{w\}\right|$. We have $d_{T}(v)=k+1$ and since $u \in N_{T}(v)-\{w\}$, we infer that $k \geq 1$. For $i \in\{1, \ldots, k\}$, let $u_{i} \in N_{T}(v)-\{w\}$ such that $u_{1}=u$. The choice of $v$ sets that

$$
u_{i} \in S(T), l_{T}\left(u_{i}\right) \geq 1 \text { and so, } u_{i} \in D \text { for all } i \text {. }
$$

Hence, by (3.1), we have $1 \leq l_{T}\left(u_{i}\right) \leq 2$ for all $i$. Assume first that $l_{T}\left(u_{j}\right)=2$ for some $j \in\{1, \ldots, k\}$. Without loss of generality, let $j=1$. Then, $u$ has another neighbor $u^{\prime \prime} \neq u^{\prime}$ in $T$. Let $T^{\prime}=T-\left\{u^{\prime \prime}\right\}$ and $D^{\prime}$ be any $\gamma_{o}\left(T^{\prime}\right)$-set. Clearly $u^{\prime}$ is the unique leaf of $u$ in $T^{\prime}$. We claim that $u \in D^{\prime}$. Suppose to the contrary that $u \notin D^{\prime}$. Then, $u^{\prime}$ and $v$ must be in $D^{\prime}$. It follows that $D^{\prime \prime}=\left(D^{\prime}-\left\{u^{\prime}\right\}\right) \cup\{u\}$ is a $\gamma_{o}(T)$-set which is different from $D$ (since $v$ belongs to $D^{\prime \prime}$ and not to $D$ ), a contradiction. This completes the proof of the claim. We have $n^{\prime}=n-1 \geq 5$. By Observation 2.2(iii), $T^{\prime}$ is an ugoa-tree. Applying our inductive hypothesis to $T^{\prime}$, we get $T^{\prime} \in \mathcal{F}$. Hence, $T$ is obtained from $T^{\prime}$ by Operation $\mathcal{O}_{1}$, implying that $T \in \mathcal{F}$. Assume now that

$$
l_{T}\left(u_{i}\right)=1 \text { and hence, } d_{T}\left(u_{i}\right)=2 \text { for all } i .
$$


For all $i \in\{1, \ldots, k\}$, let $u_{i}^{\prime}$ be the unique leaf adjacent to $u_{i}$ (with $u_{1}^{\prime}=u^{\prime}$ ). We distinguish two subcases, depending on whether $w$ belongs to $D$ or not.

Case 2.1. $w \in D$.

In view of (3.3), $T_{v}$ is a subdivided star $S S_{k}$ centered at $v$. Let $T^{\prime}=T-\left(T_{v}-\{v\}\right)$. Clearly $v \in L\left(T^{\prime}\right)$ and $w \in S\left(T^{\prime}\right)$. If $n^{\prime}=\left|V\left(T^{\prime}\right)\right|=2$, then $T$ is a wounded spider with exactly one non-subdivided edge and in this case, it is not difficult to see that such a graph is not an ugoa-tree. Hence, assume that $n^{\prime} \geq 3$. We claim the following.

If $\left|N_{T}[w] \cap D\right| \geq\left|N_{T}(w) \cap(V(T)-D)\right|+1$ with $l_{T}(w) \in\{0,1\}$, then

- when $l_{T}(w)=1, N_{T}(w)-D$ has at least one vertex $w_{t}$ such that

$$
\left|N_{T}\left(w_{t}\right) \cap D\right| \leq\left|N_{T}\left[w_{t}\right] \cap(V(T)-D)\right|+1 .
$$

- When $l_{T}(w)=0, N_{T}(w)-D$ has at least two vertices $w_{p}$ and $w_{q}$ for which (3.4) is fulfilled.

Indeed, assume first that $l_{T}(w)=1$ and suppose there is no vertex in $N_{T}(w)-D$ for which (3.4) is fulfilled. Let $w^{\prime}$ be the leaf adjacent to $w$ in $T$. In this case $(D-\{w\}) \cup\left\{w^{\prime}\right\}$ is a $\gamma_{o}(T)$-set different from $D$, a contradiction. Now, assume that $l_{T}(w)=0$. Suppose first that $N_{T}(w)-D$ has exactly one vertex, say $w^{\prime \prime}$ for which (3.4) is fulfilled. Then $(D-\{w\}) \cup\left\{w^{\prime \prime}\right\}$ is a $\gamma_{o}(T)$-set different from $D$, a contradiction. Suppose now there is no vertex in $N_{T}(w)-D$ for which (3.4) is fulfilled. Then $(D-\{w\}) \cup\{v\}$ is a $\gamma_{o}(T)$-set different from $D$, a contradiction. This complete the proof of the claim.

Observe that when $l_{T^{\prime}}(w) \in\{1,2\}$, the previous claim remains true by replacing $D$ by $D^{\prime}$ and $T$ by $T^{\prime}$. Thus, according to Observation 2.4(iii), $T^{\prime}$ is an ugoa-tree. By induction on $T^{\prime}$, we get $T^{\prime} \in \mathcal{F}$. Since $T$ is obtained from $T^{\prime}$ by using Operation $\mathcal{O}_{3}$, we directly obtain $T \in \mathcal{F}$.

Case 2.2. $w \notin D$.

By Observation 2.1(ii), $w \notin S(T)$ and so, $l_{T}(w)=0$. Since $v$ and $w$ are in $V(T)-D, v$ must have at least two neighbors in $D$. Hence, $d_{T}(v)=k+1 \geq 3$. Let $t$ be the parent of $w$, and let $X, Y$ and $Z$ be the following sets

$$
Y=C(w) \cap S(T), X=C(w)-Y \text { and } Z=D(w) \cap(S(T)-Y) .
$$

Observe that $v \in X, u \in Z, N_{T}(w)=\{t\} \cup X \cup Y$ and every vertex in $Z$ plays the same role as $u$. Therefore, by (3.2), we have $Z \subset D$ since $Z \subset S(T)$. We also have, by (3.3), every vertex in $Z$ has exactly two neighbors such that one of them is a leaf and the other one is in $X$. Furthermore, as $v \in X, u_{i} \in Z$ for all $i \in\{1, \ldots, k\}$ and so, $|Z| \geq k \geq 2$. Notice also that $|X| \geq 1$ since $v \in X$. Likewise $|Y| \geq 1$ since $D$ is a $\gamma_{o}(T)$-set. It is clear that $Y \subseteq S(T)$ and then, by Observation 2.1(ii), $Y \subseteq D$. Put

$$
X=\left\{x_{1}, x_{2}, \ldots x_{p}\right\}, \quad p \geq 1 \text {, with } x_{1}=v \text { and }|Y|=q-1, \quad q \geq 2 .
$$

The fact that every vertex in $X$ plays the same role as $v, x_{i} \in V(T)-D$ for all $i \in\{1, \ldots, p\}$, let

$$
p_{i}=\left|N_{T}\left(x_{i}\right)-\{w\}\right| \text { for } i=1, \ldots, p .
$$

We have $p_{1}=k$ and since for all $i \in\{1, \ldots, p\}, x_{i}$ and $w$ are in $V(T)-D, x_{i}$ must have at least two neighbors in $Z$. Hence, $d_{T}\left(x_{i}\right)=p_{i}+1 \geq 3$. This means that for all $i \in\{1, \ldots, p\}, V\left(T_{x_{i}}\right)$ induces a subdivided star $S S_{p_{i}}$ of order $p_{i}+1$ centered at $x_{i}$. Because $w \in V(T)-D$, inequality (1.1) is valid by replacing $v$ by $w$. This gives

$$
p \leq q-1 \text { if } t \in D \text {, or } p \leq q-3 \text { otherwise. }
$$

Let $T^{\prime}=T-\left(\cup_{i=1}^{p} T_{x_{i}}\right)$ and $D^{\prime}$ be a $\gamma_{o}\left(T^{\prime}\right)$-set. Observe that $T^{\prime}$ contains at least one $P_{3}$ as an induced subgraph, which means that $n^{\prime}=\left|V\left(T^{\prime}\right)\right| \geq 3$. For all $i \in\{1, \ldots, p\}$, let $B_{i}$ be the support vertex set of $S S_{p_{i}}$. We have $\cup_{i=1}^{p} B_{i}=Z$ and $N_{T^{\prime}}(w)=Y \cup\{t\}$, so

$$
d_{T^{\prime}}(w)=q \geq 2
$$


According to Observation 2.1(i), we can assume that $Y \subset D^{\prime}$ since $Y \subset S\left(T^{\prime}\right)$. Then, $t$ is the only neighbor of $w$ in $T^{\prime}$ that may not be in $D^{\prime}$, that is

$$
\left|N_{T^{\prime}}(w) \cap\left(V\left(T^{\prime}\right)-D^{\prime}\right)\right| \leq 1
$$

If $t \in D^{\prime}$, then the minimality of $D^{\prime}$ leads to $w \in V\left(T^{\prime}\right)-D^{\prime}$, because otherwise, we could replace $w$ by $t$ in $D^{\prime}$. By Observation 2.5(ii) and (iii), we have $D^{\prime}=D \cap V\left(T^{\prime}\right)$. Hence, $t \in D$ if and only if $t \in D^{\prime}$. Notice that if $t \in D^{\prime}$, then $N_{T^{\prime}}(w) \cap\left(V\left(T^{\prime}\right)-D^{\prime}\right)$ is an empty-set, otherwise, $t$ would be the unique vertex of $N_{T^{\prime}}(w) \cap\left(V\left(T^{\prime}\right)-D^{\prime}\right)$. Thus, (3.5) can be rewritten as follows:

$$
\text { If }\left|N_{T^{\prime}}(w) \cap\left(V\left(T^{\prime}\right)-D^{\prime}\right)\right|=0 \text {, then } p \leq q-1,
$$

and

$$
\text { if }\left|N_{T^{\prime}}(w) \cap\left(V\left(T^{\prime}\right)-D^{\prime}\right)\right|=1 \text {, then } p \leq q-3 .
$$

By Observation 2.5(iii), $T^{\prime}$ is an ugoa-tree. Applying the inductive hypothesis to $T^{\prime}$, we deduce that $T^{\prime} \in \mathcal{F}$. Since $T$ can be obtained from $T^{\prime}$ by Operation $\mathcal{O}_{4}$, we finally conclude that $T \in \mathcal{F}$. This completes the proof.

Theorem 3.3 suggests a polynomial algorithm for testing if a given tree $T$ with $n$ vertices belongs to $\mathcal{F}$ and, if so, the algorithm returns the set $A(T)$. The steps of this algorithm can be summarized as follows. If $T$ is a path on 2 or 4 vertices, answer $T \notin \mathcal{F}$ and stop. Else if $n \leq 5$, answer $T \in \mathcal{F}$, return the obvious set $A(T)$, and stop. Suppose $n \geq 6$. If $T$ has a strong support vertex, say $s$ such that $l_{T}(s) \geq 3$, call the algorithm recursively on the tree $T^{\prime}$ obtained from $T$ by removing a leaf neighbor of $s$; if the answer to the recursive call is $T^{\prime} \in \mathcal{F}$, then answer $T \in \mathcal{F}$, return $A(T)=A\left(T^{\prime}\right)$ and stop, else answer $T \notin \mathcal{F}$ and stop. Assume now that for each $x \in S(T), l_{T}(x) \leq 2$. Pick a vertex $r$, root the tree $T$ at $r$, pick a vertex $u^{\prime}$ at maximum distance from $r$. Let $u$ be the parent of $u^{\prime}$ in the rooted tree and $v$ be the parent of $u$. If $v \in S(T)$ and $l_{T}(u)=2$, call the algorithm recursively on the tree $T^{\prime}=T-T_{u}$; if the answer to the recursive call is $T^{\prime} \in \mathcal{F}$, then answer $T \in \mathcal{F}$, return $A(T)=A\left(T^{\prime}\right) \cup\{u\}$ and stop, else answer $T \notin \mathcal{F}$ and stop. If $v \in S(T)$ and $l_{T}(u)=1$, then return the answer $T \notin \mathcal{F}$ and stop. Assume now that $v \notin S(T)$. If some child of $v$ has two leaves $u^{\prime}, u^{\prime \prime}$, then call the algorithm recursively on the tree $T^{\prime}=T-\left\{u^{\prime \prime}\right\}$; if the answer to the recursive call is $T^{\prime} \in \mathcal{F}$, then answer $T \in \mathcal{F}$, return $A(T)=A\left(T^{\prime}\right)$ and stop, else answer $T \notin \mathcal{F}$ and stop. Now, assume that every child of $v$ is a support vertex of degree two. Let $w$ the parent of $v$ and further let $X=\left\{x_{1}, x_{2}, \ldots x_{p}\right\}$ and $Y$ be two subset of $V(T)$ defined as in the proof of Theorem 3.3. Assume first that $w \in S(T)$ or $|Y|<|X|$. Call the algorithm recursively on the tree $T^{\prime}=T-\left(T_{v}-\{v\}\right)$; if the answer to the recursive call is $T^{\prime} \in \mathcal{F}$ and $w$ fulfills the condition $C_{0}$ (see operation $\mathcal{O}_{3}$ ), then answer $T \in \mathcal{F}$ and return $A(T)=A\left(T^{\prime}\right) \cup B$ and stop, else answer $T \notin \mathcal{F}$ and stop. Assume now that $w \notin S(T)$ and $|Y| \geq|X|$. Call the algorithm recursively on the tree $T^{\prime}=T-\left(\cup_{i=1}^{p} T_{x_{i}}\right)$; if the answer to the recursive call is $T^{\prime} \in \mathcal{F}$, and $w \notin A\left(T^{\prime}\right)$, then answer $T \in \mathcal{F}$ and return $A(T)=A\left(T^{\prime}\right) \cup\left(\cup_{i=1}^{p} B_{i}\right)$ and stop, else answer $T \notin \mathcal{F}$ and stop.

\section{Conclusion}

In this paper, we have provided a constructive characterization of all ugoa-trees. After this work, several problems could be raised. The following seem of particular interest:

(1) Although the case of trees was solved in this paper, it is still interesting to find a characterization of all ugoa-graphs in the class of unicyclic graphs, or more generally, cactus graphs.

(2) The characterization problem of all ugoa-graphs does not seem easy, nevertheless it is an attractive point to explore.

(3) Apart from the constructive characterization of ugoa-trees, it is interesting to investigate other types of characterizations. 
(4) Knowing that any graph is not necessarily an ugoa-graph, it would therefore be interesting to characterize all graphs $G$ with the property that each vertex of $G$ belongs to at least one $\gamma_{o}(G)$-set.

(5) We can prospect another type of alliance, that is defensive alliance. A non-empty set of vertices $D \subseteq V$, of a graph $G$, is a defensive alliance of $G$, if for every $v \in D,\left|N_{G}[v] \cap D\right| \geq\left|N_{G}[v]-D\right|$. D is a global defensive alliance, or briefly a $g d a$, of $G$, if $D$ is a defensive alliance and a dominating set of $G$. An interesting problem consists to investigating all trees with a unique minimum global defensive alliance.

Acknowledgements. The authors would like to thank the anonymous referees for their valuable comments and suggestions leading to improvement of this paper.

\section{REFERENCES}

[1] H. Balakrishnan, A. Cami, N. Deo and R.D. Dutton, On the complexity of finding optimal global alliances. J. Combin. Math. Combin. Comput. 58 (2006) 23-31.

[2] M. Blidia, M. Chellali, R. Lounes and F. Maffray, Characterizations of trees with a unique minimum locating-dominating sets. J. Combin. Math. Combin. Comput. 76 (2011) 225-232.

[3] M. Bouzefrane and M. Chellali, On the global offensive alliance number of a tree. Opuscula Math. 29 (2009) $223-228$.

[4] M. Bouzefrane and M. Chellali, A note on global alliances in trees. Opuscula Math. 31 (2011) 153-158.

[5] M. Bouzefrane and S. Ouatiki, On the global offensive alliance in unicycle graphs. AKCE Int. J. Graphs Comb. 15 (2018) $72-78$.

[6] M. Chellali, Offensive alliances in bipartite graphs. J. Combin. Math. Combin. Comput. 73 (2010) 245-255.

[7] M. Chellali and T.W. Haynes, Trees with unique minimum paired domination sets. Ars Combin. 73 (2004) 3-12.

[8] M. Chellali and T.W. Haynes, A characterization of trees with unique minimum double domination sets. Util. Math. 83 (2010) $233-242$.

[9] M. Chellali and N.J. Rad, Trees with unique Roman dominating functions of minimum weight. Discrete Math. Algorithms Appl. 06 (2014) 1450038.

[10] M. Chellali and L. Volkmann, Independence and global offensive alliance in graphs. Australas. J. Combin. 47 (2010) $125-131$.

[11] O. Favaron, G. Fricke, W. Goddard, S.M. Hedetniemi, S.T. Hedetniemi, P. Kristiansen, R.C. Laskar and R.D. Skaggs, Offensive alliances in graphs. Discuss. Math. Graph Theory 24 (2004) 263-275.

[12] H. Fernau, J.A. Rodríguez-Velázquez and J.M. Sigarreta, Offensive alliances in graphs. Discrete Appl. Math. 157 (2009) $177-182$.

[13] M. Fischermann, Block graphs with unique minimum dominating sets. Discrete Math. 240 (2001) $247-251$.

[14] M. Fischermann and U.D.E. Triesch, Domination parameters and their unique realizations, Ph.D. thesis. Techn. Hochsch. Bibl. (2002).

[15] M. Fischermann and L. Volkmann, Unique minimum domination in trees. Australas. J. Combin. 25 (2002) $117-124$.

[16] M. Fischermann and L. Volkmann, Cactus graphs with unique minimum dominating sets. Util. Math. 63 (2003) $229-238$.

[17] M. Fischermann and L. Volkmann, Unique independence, upper domination and upper irredundance in graphs. J. Combin. Math. Combin. Comput. 47 (2003) 237-249.

[18] M. Fischermann, D. Rautenbach and L. Volkmann, Maximum graphs with a unique minimum dominating set. Discrete Math. 260 (2003) 197-203.

[19] M. Fischermann, L. Volkmann and I. Zverovich, Unique irredundance, domination, and independent domination in graphs. Discrete Math. 305 (2005) 190-200.

[20] M. Fraboni and N. Shank, Maximum graphs with unique minimum dominating set of size two. Australas. J. Combin. 46 (2010) 91-99.

[21] G. Gunther, B. Hartnell, L. Markus and D. Rall, Graphs with unique minimum dominating sets. In: Vol. 101 of Proc. 25th S.E. Int. Conf. Combin., Graph Theory, and Computing, Congr. Numer., Springer, New York, NY (1994) 55-63.

[22] A. Harutyunyan, Some bounds on global alliances in trees. Discrete Appl. Math. 161 (2013)1739-1746.

[23] A. Harutyunyan, Global offensive alliances in graphs and random graphs. Discrete Appl. Math. 164 (2014) $522-526$.

[24] T.W. Haynes and M.A. Henning, Trees with unique minimum total dominating sets. Discuss. Math. Graph Theory 22 (2002) 233-246.

[25] T.W. Haynes, S.T. Hedetniemi and P.J. Slater, Fundamentals of Domination in graphs. Marcel Dekker, New York, NY (1998).

[26] T.W. Haynes, S.T. Hedetniemi and P.J. Slater, Domination in Graphs: Advanced Topics. Marcel Dekker, New York, NY (1998).

[27] J. Hedetniemi, On unique minimum dominating sets in some cartesian product graphs. Discuss. Math. Graph Theory 34 (2015) 615-628.

[28] J. Hedetniemi, On unique minimum dominating sets in some repeated cartesian products. Australas. J. Combin. 62 (2015) 91-99.

[29] J. Hedetniemi, On unique realizations of domination chain parameters. J. Combin. Math. Combin. Comput. 101 (2017) 193-211. 
[30] G. Hopkins and W. Staton, Graphs with unique maximum independent sets. Discrete Math. 57 (1985) $245-251$.

[31] P. Kristiansen, S.M. Hedetniemi and S.T. Hedetniemi, Alliance in graphs. J. Comb. Math. Combin. Comput. 48 (2004) $157-177$.

[32] K. Ouazine, H. Slimani and A. Tari, Alliances in graphs: parameters, properties and applications-a survey. AKCE Int. J. Graphs Comb. 15 (2018) 115-154.

[33] N.J. Radm, A note on the global offensive alliances in graphs. Discrete Appl Math 250 (2018) 373-337.

[34] J.A. Rodríguez-Velázquez and J.M. Sigarreta, Global offensive alliances in graphs. Electron. Notes Discrete Math. 25 (2006) $157-164$.

[35] J.A. Rodríguez-Velázquez and J.M. Sigarreta, Spectral study of alliances in graphs. Discuss. Math. Graph Theory 27 (2007) $143-157$.

[36] W. Siemes, J. Topp and L. Volkmann, On unique independent sets in graphs. In Vol. 131 of Discrete Math. Elsevier, New York, NY (1994) 279-285.

[37] J. Topp, Graphs with unique minimum edge dominating sets and graphs with unique maximum independent sets of vertices. Discrete Math. 121 (1993) 199-210.

[38] I.G. Yero and J.A. Rodriguez-Velázquez, Computing global offensive alliances in Cartesian product graphs. Discrete Appl. Math. 161 (2013) 284-293. 